\title{
natureINSIGHT THE PROTEIN WORLD
}

15 September 2016 / Vol 537 / Issue No 7620

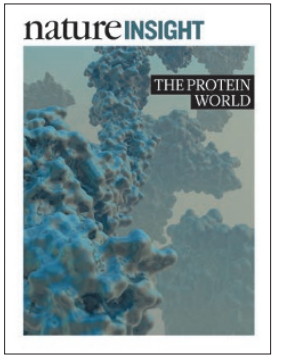

Cover illustration Nik Spencer

Editor, Nature

Philip Campbell

Publishing

Richard Hughes

Insights Editor

Ursula Weiss

Production Editor

Elizabeth Batty

Art Editor

Nik Spencer

Sponsorship

Reya Silao

Production

lan Pope

Marketing

Steven Hurst

Editorial Assistant

Giacomo Russo

The Campus

4 Crinan Street

London N1 9XW, UK

Tel: +44 (0) 2078334000

e: nature@nature.com
( ur understanding of how proteins fold into distinct shapes and catalyse a broad range of chemical transformations has grown tremendously since the term peptide was coined, more than 100 years ago. Here, we present a collection of reviews that highlight four of the most exciting topics that are being investigated by contemporary protein scientists.

State-of-the art computational methods can now be used to design non-natural proteins that show promise as therapeutic agents and nanomaterials from scratch. David Baker and his colleagues describe how computers can be used to accurately generate de novo designed proteins that assemble into desired, defined shapes.

The proteome - a collection of all proteins that are expressed in a cell in a specific context - is extremely complex, especially given that some proteins exist in several states. Wade Harper and Eric Bennett discuss how cells monitor and regulate the relative abundance of each protein in the proteome through quality control mechanisms. They also explore how this knowledge can be used to improve understanding of the function of cells and to develop potential therapeutic drugs.

Cryo-electron microscopy is in the middle of a revolution (Nature 525, 172-174; 2015), and now the technique is being used routinely to obtain highresolution structures of proteins and protein complexes. Rafael Fernandez-Leiro and Sjors Scheres describe the research that led to this revolution and illustrate how powerful the method has become, especially with respect to membrane proteins and large protein complexes.

The most effective way to perform large-scale measurements of protein complexes and proteomes is to use high-resolution mass spectrometry, with advances in technology enabling almost complete proteomes to be analysed. Ruedi Aebersold and Matthias Mann explain how the technique is being used to catalogue the components of proteomes and their sites of posttranslational modification, to identify networks of interacting proteins and to uncover alterations in the proteome that are associated with diseases.

We hope that this collection will inspire the next generation of biochemists, biophysicists and molecular biologists to explore the many uncharted regions of the protein world.

Joshua Finkelstein, Alex Eccleston \& Sadaf Shadan Senior Editors

\section{CONTENTS}

\section{REVIEWS}

320 The coming of age of de novo protein design

Po-Ssu Huang, Scott E. Boyken \& David Baker

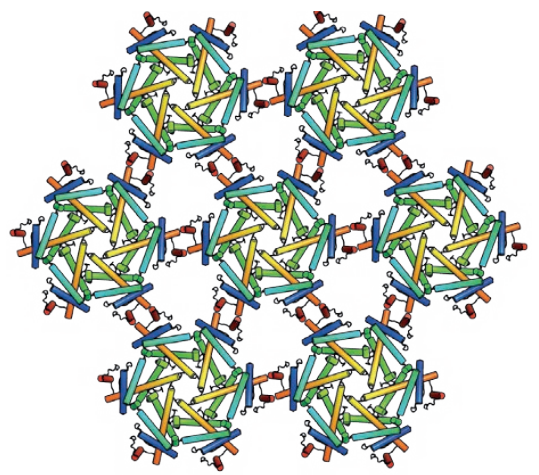

328 Proteome complexity and the forces that drive proteome imbalance J. Wade Harper \& Eric J. Bennett

339 Unravelling biological macromolecules with cryo-electron microscopy

Rafael Fernandez-Leiro \& Sjors H. W. Scheres

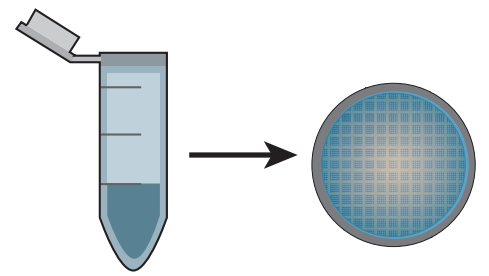

347 Mass-spectrometric exploration of proteome structure and function Ruedi Aebersold \& Matthias Mann

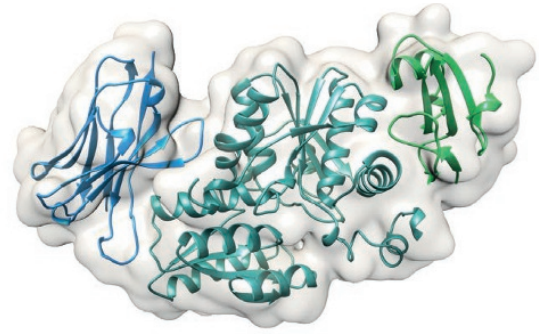

